\title{
HANDOVER MANAGEMENT SCHEME IN LTE FEMTOCELL NETWORKS
}

\author{
Tijane Fatima Zohra BADRI ${ }^{1,2}$, SAADANE Rachid $^{1}$,Mohammed Wahbi ${ }^{1}$ \\ and MbarkiSamir ${ }^{2}$ \\ ${ }^{1}$ Electrical Engineering DepartmentSIR2C2S/LASI-EHTP Casablanca, morocco \\ ${ }^{2}$ Departement of Computer Science, Faculty of Science LARIT, Ibn Tofail \\ UniversityKenitra, morocco \\ ${ }^{1}$ tijanebadri@hotmail.fr \\ ${ }^{2}$ Rachid.saadanelgmail.com \\ ${ }^{3}$ wahbi_mho@yahoo.fr \\ ${ }^{4}$ mbarkisamirahotmail. com
}

\begin{abstract}
Femtocell is a small cellular base station in home, a low-power wireless access point designed for use in residential or small business environment, this technology is a promising solution for operators to improve network performance, and the deployment of a femtocell is a key for bandwidth limitation and coverage issues in conventional mobile network system. Therefore, the inter-cell handover process will become more complex, frequent and time-sensitive with the introduction of femtocells in cellular networks. This paper presents the architecture of LTE femtocell networks and investigates the different scenarios in the handover procedure. Especially, the mobility of user from macrocell to femtocell which is quite difficult due to the large number of candidate femtocells in the coverage area and the characteristics of the femtocell entity, In order to achieve the optimize procedure handover decision policy based on using the advantage of the entity HeNB Policy Function to select and make decision of handover based on different constraint to make the optimal decision about the target femtocell to mitigate the unnecessary handovers, hence we describe the decision policies rules as well as the handover signalling flow furthermore, an analytical model was presented for the handover signalling cost.
\end{abstract}

\section{KEYWORDS}

Handover, femtocell, HeNB, macrocell, HeNB policy function, LTE.

\section{INTRODUCTION}

The concept of mobility offer several advantages to users, they can stay connected by handovers to the cells closer by as they move in the network all the while maintaining their services. Seamless mobility and anywhere anytime type of service provision, have always been key design principles for legacy cellular networks, in this work we focus on the mobility management in LTE based femtocell network.

Femtocell is a small cellular base station designed for use in subscriber's home and small business environments. It's the emerging network technology which is defined broadly as lowcost, low-power cellular base stations [1]. 
The femtocell concept can be applied on different radio access technologies it has been discussed in Long Term Evolution LTE system by the name of Home e-NodeB (HeNB), it also known as femto access point FAP a main device in femtocell network that provides radio access network RAN functionality.

The network management and integration of femtocell with LTE macrocell networks is different from the existing LTE networks. Thousands of femtocells inside a macrocell area create interference problem, also due to huge number of possible target femtocell candidates for macrocell to femtocell handover, a large neighbor list and communication with many femtocells for the pre-handover procedure are needed, which may cause signalling overhead and unnecessary handovers. The optimal solution of these two problems can improve the performance of femtocell networks [2]. In other hand, UEs with high velocities moving through the femtocell usually lead to performing some unnecessary handovers, where an outbound handover happens quickly after an inbound handover, these cause a decreasement of the network performance. Otherwise, another important parameter used in the purpose to protect the rights of the users who equip a HeNB in their own house and pay for it, the definition of closed subscriber Group (CSG) is proposed in [3]. It identifiessubscribers of an operator who are permitted to access one ormore femtocells of E-UTRAN but which have restrictedaccess (CSG cells). The definition of CSG generates threeaccess modes which allow users to access femtocells, the open access mode, CSG access mode and the hybrid access mode.

The open mode designed for public access, the CSG access mode each UE that is not the member of the CSG is not allowed to access CSG HeNBs noted that UEs hold a whitelist in the USIM containing a user controlled list of the allowed CSG identities, finally the Hybrid access same as CSG mode in addition that an UE that is not part of the CSG may camp and receive some level of service from the HeNB.

In this paper we overview the architecture of LTE femtocell networks and investigate the different scenarios in the handover procedure specially, the mobility of user from macrocell to femtocell which is quite difficult due to the large number of candidate femtocells in the coverage area and the characteristics of the femtocell entity. The handover decision policy is optimized by using the benefit of the entity HeNBPolicy Function to select and make decision of handover based on different constraint to make the optimal choice about the target femtocell.

The rest of this paper is organized as follow: In section two we present some research works in handover management of femtocell networks, in section three we describe the femtocell system architecture in E-UTRAN, in section four we overview the architecture of LTE femtocell networks and investigate communication between femtocells and macrocells the different scenarios in the handover procedure as well as the handover management with the decision policies rules used to select the optimal target femtocell, moreover the signalling flow for the Hand-in handover procedure finally an analytical model was presented for the handover signalling cost evaluation.

\section{RELATED WORK}

In femtocell network, several research works have been published. In [5], it proposed a mobility management scheme that move the mobility anchor for user plane from the S-GW to the HeNB GW and let the HeNB make the handover decision in HeNB- HeNB handover scenario.

The authors in [3] are introduced a call admission control optimization algorithm based on velocity and the real-timing attribute of the user's service for femtocell network, However, the algorithm involved the detection and the judgment of the real timing attribute, which is complicated and not suitable for a cost-effective implementation. 
In [4] it proposed a new Autonomic Architecture with Self-organizing capabilities based on the election of a Femtocell cluster Head (FH) for each group of Femtocell APs. The FH will be responsible to dynamically adjust the network overall coverage to save FAP energy and provide better QoS to users.

More detail works on handover in femtocell network, in [6], it proposed a handover decision algorithm that combined the values of received signal strength from a serving macrocell and a target femtocell in the consideration of large asymmetry in their transmit powers in eNB-toHeNB handover scenario.

\section{FEMTOCELL SYSTEM ARCHITECTURE IN E-UTRAN}

The network architecture of LTE system consists of macrocells with eNodeBs on providing both user plane and control plane to the UEs, femtocells being a new addition to the existing components. The eNodeBs are interconnected with each other by the $\mathrm{X} 2$ interface which is mainly used for inter-eNodeB handover purpose and also connected to the Mobility Management Entity MME which the functions is related to handover and the Serving Gateway (S-GW).The Mobility Management Entity MME is the key control node for the LTE access network that processes the signalling between the $\mathrm{UE}$ and the $\mathrm{CN}$, It is also responsible for authenticating the users and for the generation and allocation of temporary identities to UEs, the MME also terminates the S6a interface toward the home HSS for roaming UEs, also handles control plane signalling, especially for mobility management. The S-GW is mobility anchoring for the inter$3 \mathrm{GPP}$ mobility and routes and forwards the packet.

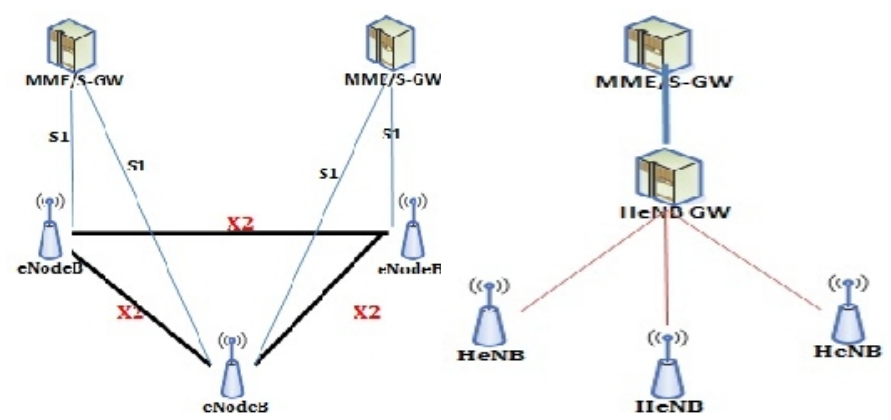

Figure 1. The E-UTRAN architecture and the femtocell architecture in E-UTRAN.

As hundreds of thousands of femtocells are deployed, the scalability issue imposes costly reconfiguration and operation in MME/S-GW because femtocells use residential broadband as the backhaul to connect to the mobile core network $(\mathrm{CN})$, security issue needs be considered in order to protect the integrity of the network from malicious operations. Therefore, the femtocell network needs to consider both of the problems. Figure 1 shows femtocell E-UTRAN architecture where femtocell is referred to as Home eNB (HeNB). An intermediate node called HeNB Gateway is proposed to be located between HeNBs and the mobile CN. It acts as a "virtual" macro eNodeB towards CN and as a "virtual" CN node towards the HeNBs. Hundreds of HeNBs are connected to the EPC through the HeNB GW; this one should appear to the MME as an eNodeB and appear to the HeNB as an MME between the HeNB and the Core Network. The interface between every HeNB $\leftrightarrow$ HeNB GW and each HeNB GW $\leftrightarrow$ MME/S-GW is S1 interface, no X2 interface exists between neighboring HeNBs [5]. 
The figure 2 shows the logical architecture of LTE femtocell in 3GPP, which involve the entity HeNB GW acting as the role of concentrator, The HeNB GW entity is assigned with a normal eNodeB ID (a unique identity for an eNodeB among nodes in E-UTRAN) in the purpose to be seen by the MME as any other eNodeBs. The HeNB GW can allocate private identities for each HeNB within its range and maintain a list of these HeNB IDs, the architecture contain also the HeNB PF for selecting the target femtocell.

The most acting elements in the femtocell logical architecture are the security Gateway (SeGW) who's responsible for the authentication of femtocell, and providing the femtocell with access to the HeNB GW, this latter terminates S1 from femtocell and appears as an RNC to the existing core network using S1 interface, supports femtocell registration and UE registration over S1, and the HeNB entity provides RAN connectivity using the S1 interface, supports RNC functions, HeNB registration and also supports UE registration over S1 [7]. In other side we have the HeNB Policy Function HeNB PF which responsible for making decisions according to the characteristics of HeNB about whether the admission quest can be accepted or rejected interacts with other policy entity like PCRF [8].

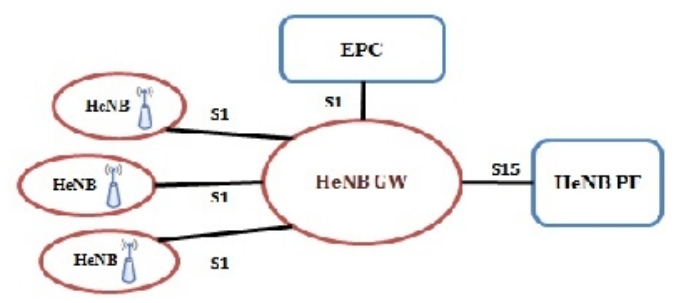

Figure 2. Logical architecture for femtocell network.

\section{LTE HANDOVER PEROCEDURE AND SIGNALLING FLOW}

The handovers allows communication during user's movement in the network they are used to keep mobile clients connected to their service network, even when these clients roam from a network access point to another network access point, so this procedure is very important for mobility support in wireless networks.

In LTE network, the handover decision between two eNodeBs are made by the eNodeBs entity itself without consulting with the MME: the source eNodeB decides to move the UE to the target eNodeB based on UE measurement report, the target eNodeB prepares radio resources before accepting the handover, after a successful handover, the target eNodeB indicates the source eNodeB to release the resources and sends a path switch message to the MME. The latter sends a User Plane Update Request to the S-GW about to which eNodeB the packets for the user shall route. The control messages are exchanged via the X2 interface between the two eNodeBs, and the downlink packet data is also forwarded from the source to the target eNodeBvia the same X2 interface [7][8]. The figure 3 illustrates the components of the LTE network architecture with femtocell integration.

In our procedure, we make use of the entity HeNB PF to make decisions taking the special elements of femtocell into consideration, user type, access mode, speed status, large amount, small coverage and radio resource control...The aim of using this mechanism to select the target femtocell is to avoid some unnecessary handovers which present an important requirement for an efficient handover mechanism. The lower the number of unnecessary handovers the more efficient the handover mechanism is. 


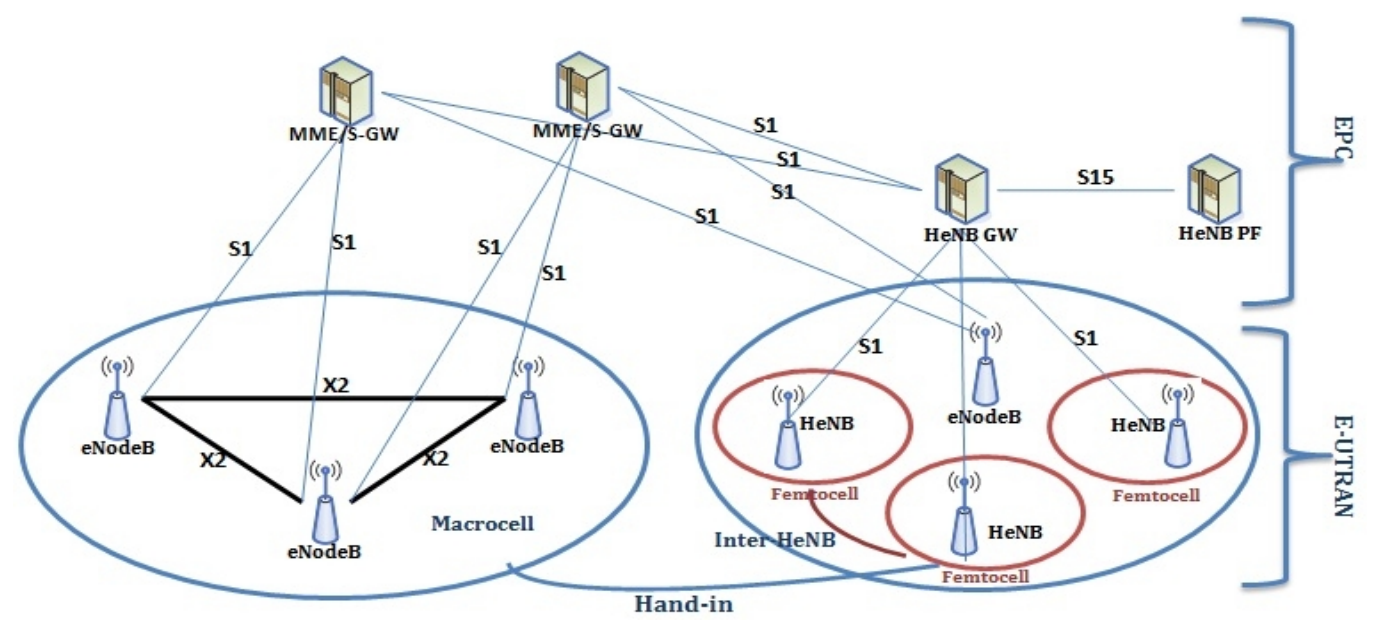

Figure 3. The overall LTE system architecture with deployed HeNB.

\subsection{Handover scenario in femtocell}

The handover procedure allows communication during user's movement among the network, it is critical to support the user's mobility in all current mobile systems including the femtocell network. The handover procedure in femtocell network contains three scenarios [5]:

\subsubsection{Hand-in procedure (macrocell $\leftrightarrow$ femtocell)}

Represents the handover scenario where a UE switch out from macrocelleNodeB to HeNB, this scenario is a difficult procedure and quite demanding since there are hundreds of possible targets HeNBs. In hand-in procedure, the UE needs to select the best target HeNB so the optimal handover decision policy is so critical and difficult, so we take advantage of the entity HeNB PF to select the most appropriate target HeNB according to the predefined rules(figure 4).

\subsubsection{Hand-off procedure (femtocell $\leftrightarrow$ macrocell)}

The handover that is performed from HeNB to macrocelleNodeB, it is not so complicated as the hand-in, because there is only one candidate and UE is no need to select the optimal target cell or to use the HeNB PF entity. That is to say that complex target cell selection mechanism is unnecessary. When the signal strength from the macrocell is higher than the one from serving $\mathrm{HeNB}$, the UE will connect to it and transmit the data packets using the target macrocelleNodeB without considering so many elements as those in the hand-in decision making phase.

\subsubsection{Inter-HeNB procedure (femtocell $\leftrightarrow$ femtocell)}

Represents the interaction between two HeNBs, the handover from one HeNB to another HeNB in the same macrocell network. The inter-HeNB handover is similar to hand-in procedure since in this scenario, there are still hundreds of candidate target HeNBs when UE move out the coverage of its serving HeNB. So in this scenario the target cell selection mechanism is necessary an appropriate selection for the efficient handover is required. Therefore, in this situation we need to use HeNB PF to select the optimal target HeNB. 


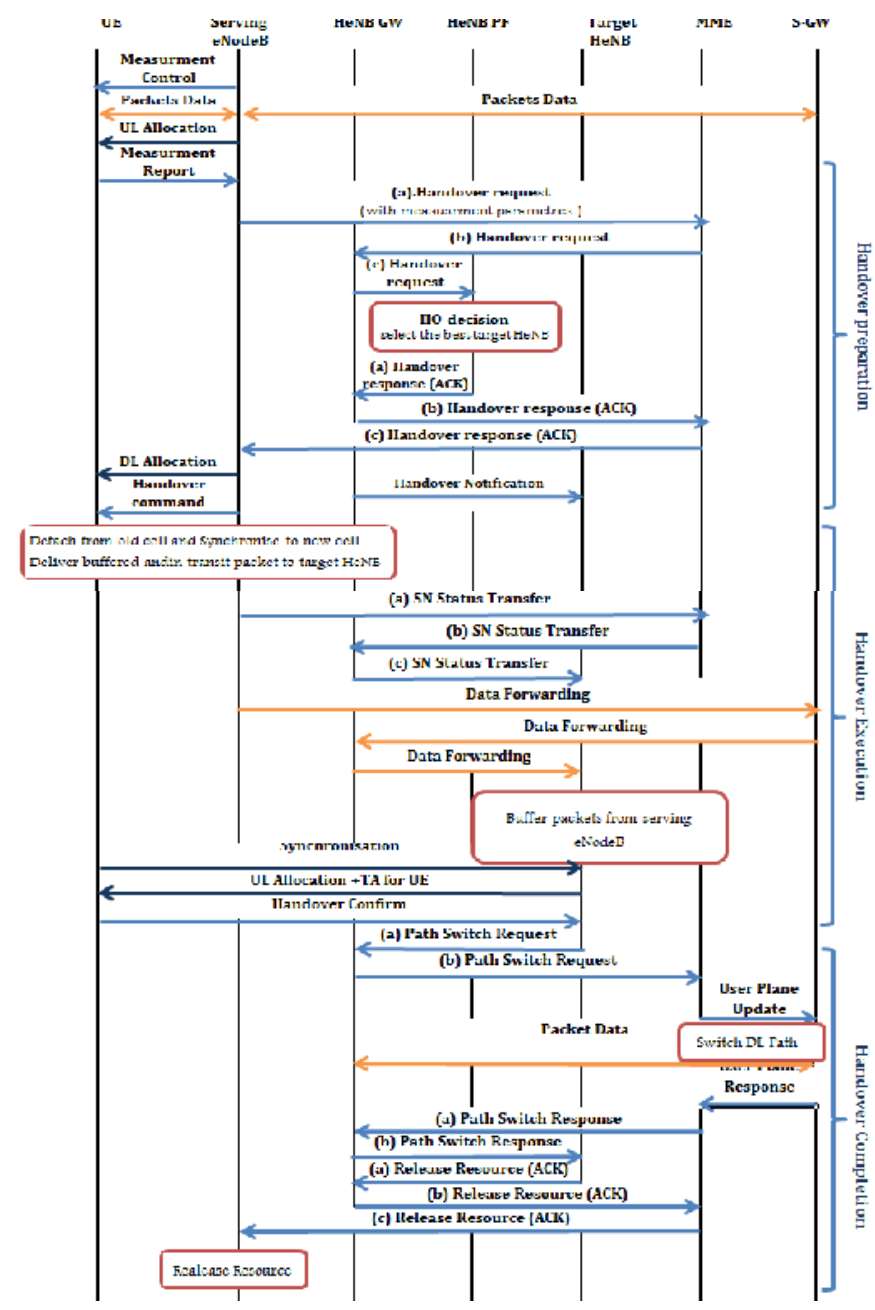

Figure 4.Signalling flow of Hand-in procedure.

\subsection{Handover management}

The development of solutions for mobility scenarios is the challenging issue in the handover management, this latter present the process by which mobile terminal maintains its connection active while moving from one access point to another.Seamless mobility across radio technologies with deployment of the HeNB is complicated to implement therefore, the ability to seamlessly switch between the femtocell and the macrocell networks is a key driver for femtocell network deployment.

The handover procedure is mainly divided into three phases: handover information gathering phase, handover decision, and handover execution phase. The UE collects information about the handover candidates and during the handover decision phase, the best handover candidate is determined, after deciding to perform the actual handover, the UE initiates to connect with new FAP. Otherwise, the biggest challenge in the handover is to select the best target femtocell from so many candidates. Especially, considering the different characteristics of femtocell, like its access mode and radio resource control function, the selection and the decision of which FAP is hard to make. 
The handover decision is the most important phase in the handover procedure which contains measurements and information about when and where to perform handover and obtained from one entity or more [4]. We are concerned by the handover decision method to develop the handover policies in order to optimize the selection of the optimal target femtocell in a way that multiple criteria from terminal and network sides and advanced decision is needed, this system is integrated into the HeNB PF entity.

-Handover Information Gathering. Responsible for collecting all the contextual information, through monitoring and measurements, require to identify the need for handover and to apply handover decision policies.

- Handover Decision. Defining all requires for the handover (policies) and how to perform it by selecting the most optimal Femtocell based on decision parameters.

- Handover Execution. This means establishing the IP connectivity through the target access femtocell. For that, we can use fast Mobile IP functionalities as an IP mobility management solution.

\subsection{Femtocell selection}

\subsubsection{Handover Policies}

The decision policy rules represent a group of parameters and instructions translate scenarios related to connectivity, network availability user, or even corporate preferences, in order to be used in femtocell selection step.

The Handover Policies defined as follows: "if condition then action" where the action part is triggered when the condition part is satisfied [4]. For selecting the appropriate femtocell target we must have some constraint, so we need more decision criteria from the user side as well as from the network. The most suitable femtocell, from those available, has to be selected satisfying a number of objectives.

So, we consider the most dual decisive objectives: The mobility state of UE (speed status) and CSG deployment (CSG ID), a decision making method in which all available alternatives femtocells are evaluated according to these objectives.

- Mobility state of UE.The states of UE speed should be considered to manage the mobility, to reduce the frequent handover trigger, the ping-ponging effect, so the mobility state of UE effects directly the handover decision.

The traditional handover algorithmlet the high speed macrocell user's handover to the femtocell,which may introduce two times unnecessary handovers forthe user. Otherwise, the high speed macrocell users usually do not want tohandover to the femtocell while the low speed may be want tohandover to the femtocell but they must satisfying another important criteria the access mode of the femtocell.

- Access mode of HeNBinfemtocell network. The HeNB is connected to the EPC through an insecure link, which brings new risks to the LTE network management, the access mode of HeNB may bring a result that UE isn't permitted to access the target HeNB selected by serving eNodeB moreover, the lack of radio resources in the selected cell may cause handover failure too, there are three types of access mode for the HeNB. The open access mode has no access limits for the users, the functions supported by a HeNB are similar to those supported by an eNB and the procedures executed between a HeNB and an EPC are the same as those between an eNB and the 
EPC so in this mode, if any User Equipment (UE) receives a higher signal level from a specific femtocell than that from a specific macrocell, it can access the femtocell as long as there is surplus bandwidth.

In the CSG access mode, only the CSG users can be authorized to access it, because the UEs hold a whitelist in the USIM containing a user controlled list of the allowed CSG identities. The hybrid access, this mode is similar to the CSG with the exception that an UE that is not part of the CSG may camp and receive some level of service from the HeNB, so each femtocell has its CSG users who are able to access it [9].

Actually, the eNodeB entity can't obtain such information as access mode and current load of the possible target HeNBs because there is no interface between them in the current architecture.

In order to optimize the efficient of the handover procedure we turn the job of selecting target femtocell over to the HeNB PF entity which can interact with the HeNBs and get the HeNB special information. In our scheme, the HeNB PF entity uses two aspects of information as input to make decisions. One is the measurement parameters (Received Signal Strength Indication RSSI and Interference -and-Noise Ratio INR...) contained in the handover requests and the other is the HeNB special parameters through the interactions with HeNBs, like its CSG ID, access mode, speed status, current load, capability and other parameters.

The HeNB PF entity use some decision rules for selecting the appreciate target based on [9]. When the HeNB PF receives the handover requests containing the measurement parameters it means the HeNBs' RSSI and INR whose RSSI is stronger than the serving eNodeB's, it obtains those HeNBs' CSG IDs, the access mode, and current load. Then, it will contrast them with the Allowed CSG List of the UE. We supposed that the reported HeNBs' RSSIs are slightly different with others. So this will generate two results [9]:

1) There are some HeNBs whose CSG IDs are in the allowed CSG list. In this case, there are five kinds of target HeNBs.

2) There is no candidate HeNBs which can match with the list. In this case, there are only three types of target HeNBs.

The HeNB PF entity will give different priorities to them, the priority order from high to low.

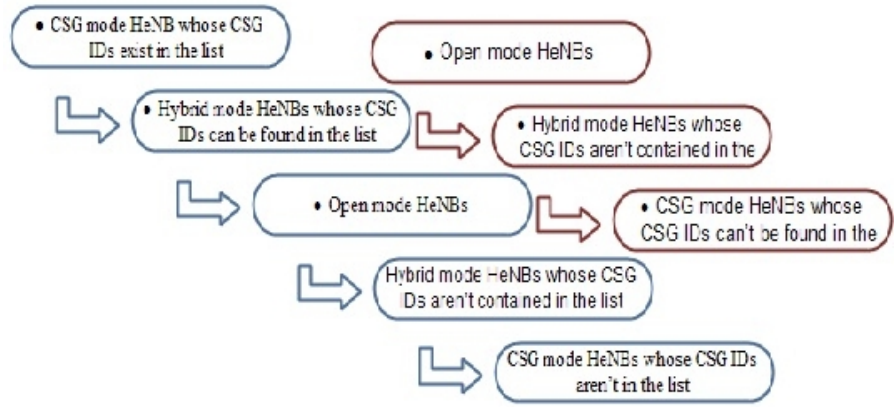

Figure 5. The setting priority in HeNB PF.

\subsubsection{The Decision Making}

After receiving the speed status of UE, all users with high mobility no handover to perform, to avoid the ping-ponging effect, the UE moves from macrocell to femtocell and within an extremely short time it moves to macrocell again otherwise, the low macrocell users are preferred to handover into femtocell in this case, the HeNB PF entity applied the second parameter to select the target HeNB using the access mode of the possible target femtocell. 
The HeNB PF chooses the optimal target HeNBs after a successful setting priority for the possible targets and favors the one with higher priority. When all the higher priority HeNBs can't admit the UE resulted from having no surplus bandwidth, lower priority HeNBs will be considered. We may face with such a situation that multiple HeNBs with same priority exist simultaneously. This problem can be addressed by comparing their RSSI and current load and the one with higher signalling strength and smaller load will be selected. The worst-case scenario is that there is no appropriate HeNB to handover to, in this case the HeNB PF will send handover failure notification with the reasons that can't find the appreciate target HeNB to the MME [9].

The HeNB PF entity help to reduce the probability of handover failure as the target HeNB is selected based on many factors such as user type, speed status of UE, HeNB access mode, current load and signalling strength, handover failure caused by access limitation or heavy load won't occur.

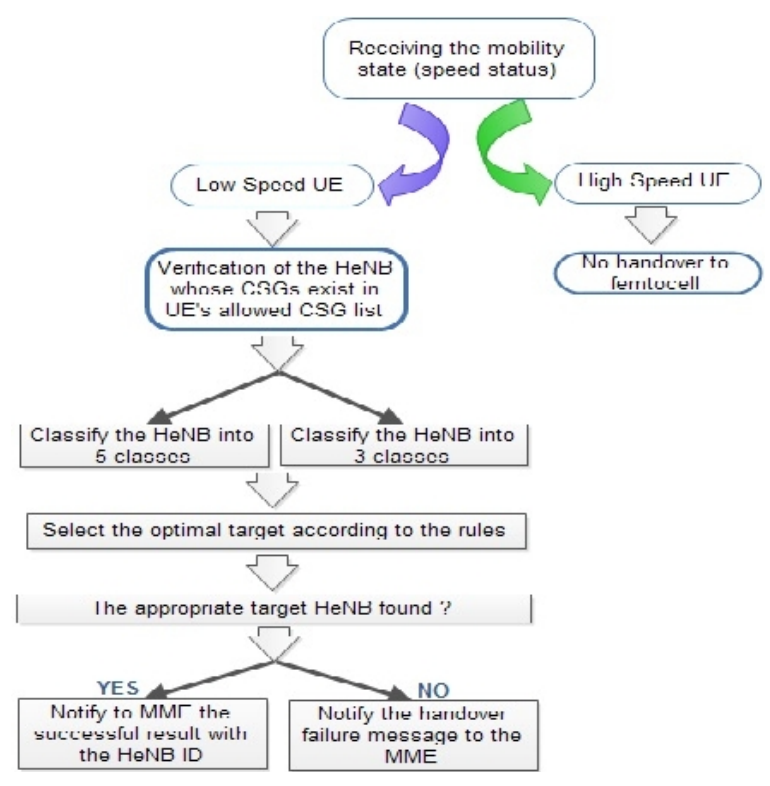

Figure 6. The operation of decision in HeNB PF

\section{ANAL YTICAL MODEL}

Now in this section we present a simple analytical model to investigate the signalling cost based on the work in [8] [10].The signalling cost for the handover in case of macrocell into femtocell.

The mobility patterns of an UE influence the number of handover in a handover scenario, therefore it can determine the average signalling cost of each handover scenario, and we assume that there are two mobility state of the UE, low mobility state like slow walk, stationary and the high mobility state for UE in train or drive a car...

Let $\alpha$ denotes the proportion of the high mobility users while $1-\alpha$ means the proportion of the low mobility users.

The high proportion of high mobility users is depicted by the big value of $\alpha$ although, low $\alpha$ represents a high proportion of low mobility users. In case of handover between macrocell and femtocell two scenarios are considered. 
Scenario 1: an UE in active state moves into and ends the active state in a femtocell whose session initializes in the macrocell.

Scenario 2: an UE in active state moves across the femtocell, whose session initializes in the neighbor macrocell, and finally moves out of the femtocell.

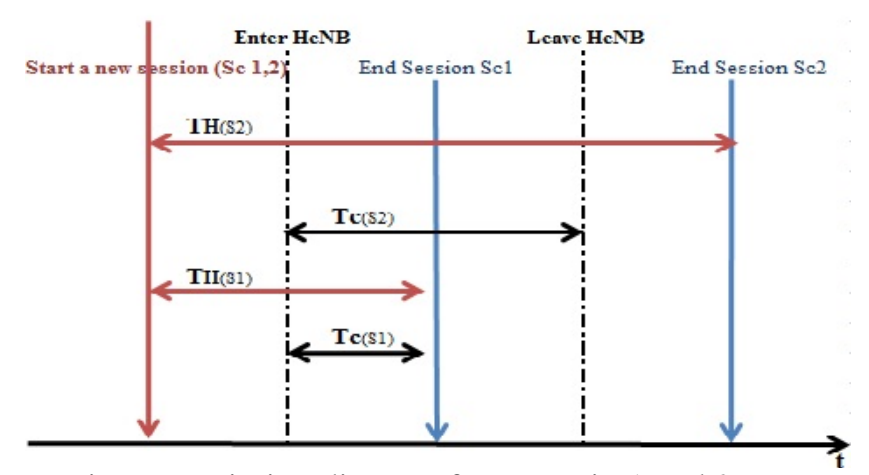

Figure 7. Timing diagram for scenario 1 and 2.

\subsection{The probability of handover}

We must define some parameters in order to define the probability of handover in each scenario as discussed in [11] [12].

The UE's camp time in the femtocell represent the time from an active UE moves into the femtocell until the time it stops in the femtocell and is denoted by Tc1 in scenario 1 .

Tc2 is denoted as the UE's camp time in the femtocell in scenario 2, which is an active UE who initializes the session out of the femtocell comes into the femtocell to the time it leaves the femtocell, while TH1 and TH2 designed the session holding time in scenario 1 and scenario 2 respectively. Otherwise, the probability distribution function of low mobility users camp time in femtocell is $f_{F L}$ with the pdf $\mathrm{K} / \mathrm{t}$ ( $\mathrm{K}$ is the standardization factor, $\mathrm{t}$ is the camp time of the low speed user in the femtocell), in other hand, the $f_{F H}$ signify the probability distribution function of high mobility users camp time TFH.

If we denote the high mobility users macrocell camp time as $T_{M H}$ with the pdf $f_{M H}$ which is assumed exponentially distributed with mean $1 / \mu, \mathrm{r}$ and $\mathrm{R}$ are the radius of the femtocell and macrocell, respectively, we assume also that the user has a constant speed $v(\mathrm{~m} / \mathrm{s})$ in the femtocell and macrocell that is:

$$
\begin{gathered}
v=T_{F H} / \mathrm{r}=T_{M H} / \mathrm{R} \\
T_{F H}=\left(\frac{r}{R}\right) T_{M H}
\end{gathered}
$$

Then, we can get the following equation for the probability distribution function of high and low mobility user's camp time [10].

$$
f_{F H}=\left(\frac{r}{R}\right) f_{M H}
$$

Let $\mathrm{T}_{\mathrm{F}}=\mathrm{T}_{\mathrm{FH}}+\mathrm{T}_{\mathrm{FL}}$ denotes the camp time of both high mobility and low mobility users in femtocell with the $\operatorname{pdf} f_{F}(t)$, [10] given by: 


$$
\begin{aligned}
& \mathrm{T}_{\mathrm{F}}(\mathrm{t})=\alpha \mathrm{T}_{\mathrm{FH}}(\mathrm{t})+(1-\alpha) \mathrm{T}_{\mathrm{FL}}(\mathrm{t}) \\
& \mathrm{f}_{\mathrm{F}}(\mathrm{t})=\alpha\left(\frac{\mathrm{r}}{\mathrm{R}}\right) \mathrm{f}_{\mathrm{MH}}(\mathrm{t})+(1-\alpha) \mathrm{f}_{\mathrm{FL}}(\mathrm{t})
\end{aligned}
$$

The form expression of Psiand Psacan be derived as follows [10], where, $\mathrm{U}$ is the upper limitation of the femtocell users' camp time:

$$
\begin{gathered}
\text { Ps } 1=\alpha \frac{\mathrm{R}}{\mathrm{r} \varepsilon+\mathrm{R}}+(1-\alpha)+\frac{\mathrm{e}^{\varepsilon}+\mathrm{KEi}(-\varepsilon)}{\varepsilon \mathrm{K}(\mathrm{U}-1)} \\
\text { Ps } 2=\alpha \frac{\mathrm{R}}{\mathrm{r} \varepsilon+\mathrm{R}}+(1-\alpha)+\mathrm{KEi}(-\varepsilon) \\
\text { Where, } \quad \operatorname{Ei}(\mathrm{x})=\int_{-\mathrm{x}}^{\infty} \frac{1}{\mathrm{ye} \mathrm{y}} \mathrm{dy}
\end{gathered}
$$

$\mathrm{TheF}_{\mathrm{T}_{\mathrm{H}}}(\mathrm{x})$ is the cumulative distribution function of $\mathrm{T}_{\mathrm{H}}$ where $\mathrm{i}=1,2$, and $\mathrm{T}_{\mathrm{H}}$ follows the exponentially distributed with the mean of $\frac{1}{\varepsilon}, \mathrm{P}_{\mathrm{H}}^{\mathrm{si}}$ and $\mathrm{P}_{\mathrm{L}}^{\mathrm{si}}$ are the probability of high speed users and low speed users in scenario $\mathrm{i}(\mathrm{i}=1,2)$ respectively.

\subsubsection{The probability of selecting a CSG cell not allowed to access}

The probability Ps ofselecting a CSG cell not allowed to access is calculated asfollows [9]:

$$
\text { Pns }=\text { Pc } * \text { Pncsg/c }
$$

With Pc represent the probability that the selected cell is a CSG mode HeNB $0<\mathrm{Pc}<1$, and the Pncsg/c signify the probability that the CSG ID of the selected CSG mode HeNB isn't in UE's Allowed CSG list. $0<P n c s g / c<1$, as we will never select the CSG cell whose CSG ID isn't in UE's Allowed CSG list in our proposal, so Pncsg/c=0 in our scheme.

\subsubsection{The probability of selecting a cell which can offer better QoS for UE.}

The cells that can offer better QoS for the UE refers to CSGcells whose CSG ID is in UE's Allowed CSG list and hybridcells whose CSG ID is in UE's Allowed CSG list. So theprobability of selecting a cell which can offer better QoS forUE can be calculated through the expression below [9]:

$$
\mathrm{Ps}=\mathrm{Ph} * \mathrm{Pcsg} / \mathrm{h}+\mathrm{Pc} * \mathrm{Pcsg} / \mathrm{c}
$$

With the probability $\mathrm{Ph}$ is that the selected cell is a hybrid mode $\mathrm{HeNB}, 0<\mathrm{Ph}<1$, otherwise the $\mathrm{Pc}$ is probability that the selected cell is a CSG mode HeNB, $0<\mathrm{Pc}<1$, for the Pcsg/h present the probability that the CSG ID of the selected hybrid mode HeNB is in UE's Allowed CSG list, $0<\mathrm{Pcsg} / \mathrm{h}<1$.

The probability of the handover happens between the macrocell and femtocell is the sum probability of scenario 1 Ps 1 and scenario 2 Ps 2 with the sum probability of selecting a CSG cell not allowed to access and selecting a cell which can offer better QoS for UE.

$$
\mathrm{PH}=(\mathrm{Ps} 1+\mathrm{Ps} 2)^{*}(\mathrm{Ps}+\mathrm{Pns})
$$




\subsection{Cost signalling evaluation}

The handover signalling can be divided into transmitting signalling and processing signalling. So the transmission cost is the cost of delivering handover message between two nodes and processing cost is the cost of processing messages at each node in the network. The signalling cost of the HeNB related handover in each scenario is derived as follows:

$\mathrm{C}=\mathrm{P}_{\mathrm{H}}\left(\sum \mathrm{T}_{\mathrm{j}}^{\mathrm{i}}+\sum \mathrm{P}_{\mathrm{k}}\right)(10)$

Where $\mathrm{P}_{\mathrm{H}}$ is the probability of the handover in a scenario, and $\left(\sum \mathrm{T}_{\mathrm{j}}^{\mathrm{i}}+\sum \mathrm{P}_{\mathrm{k}}\right)$ is the signalling cost. The signalling cost associated with each handover that is occurred when UE crosses border of two (H) eNBs in active mode is the sum of the transmission cost $T_{j}^{i}$ and processing $\operatorname{cost} P_{i}$, where $i$ and $j$ indicate nodes in the network, and pair $(i, j)$ indicates the two nodes exchanging the signalling messages between each other, such as (HeNB, HeNB GW) or (HeNB GW, CN).

\section{CONCLUSION}

The integration and management of femto-cellular networks with macro-cellular networks present the crucial part for the successful deployment of the femtocell technology. However the macrocell into femtocell handover procedure is the most demanding and difficult process, beside it is a challenge to make a selection and decision of the target HeNB, to address this issue, we intend to use the HeNB PF that selects the optimal target HeNB based on the information included in the measurement reports and the special parameters obtained from HeNBs, especially the speed status of the UE and the CSG access mode of the Target HeNB in order to protect the rights of the users.

\section{REFERENCES}

[1] V. Chandrasekhar, J. Andrews, "FemtocellNetwoks : Survey," IEEE Communication Magazine, Vol.46, No.9, pp59-67, Sept.2008.

[2] Jin-SeokKim,Tae-Jin Lee , "Handover in UMTS Networks with Hybrid Access Femtocells", the 12th International Conference Advanced Communication Technology (ICACT), vol.1, pp.904-907, 7-10 Feb.2010.

[3] 3GPP TS 25.367 V9.3.0 (2010-03). "Mobility procedures for Home Node B (HNB); Stage 2."

[4] K.Sethom Ben Reguiga F. Mhiri ,R.Bouallegue., "Handoff Management in Green FEMTOCELL Network", International Journal of Computer Applications (0975 - 8887)Volume 27- No.4, August 2011

[5] L. Wang, Y. Zhang, and Z. Wei, "Mobility management schemes at radio network layer for LTE femtocells," in IEEE VTC'09, pp. 1-5.

[6] J. M. Moon and D. H. Cho, "Novel handoff decision algorithm in hierarchical macro femto-cell networks," in IEEE WCNC'09.

[7] 3GPP TS 25.467 V9.2.0 (2010-03), "UTRAN architecture for 3G Home Node B (HNB); Stage 2

[8] 3GPP TR 23.839 V0.1.1 (2010-05), "Study on Support of BBF Access Interworking"

[9] TiantianBai, Yuwei Wang, Yu Liu, Lin ZhangA "Policy-Based Handover Mechanism between Femtocell and Macrocell for LTE based Networks" 978-1-61284-307-0/11/\$26.00 (C2011 IEEE.

[10] Haijun Zhang, Wenmin Ma, Wei Li, Wei Zheng, Xiangming Wen Chunxiao Jiang "Signalling Cost Evaluation of Handover Management Schemes in LTE-Advanced Femtocell" 978-1-4244-83297/11/\$26.00 @2011 IEEE

[11] J.S.M. Ho and I. F. Akyildiz, "Local Anchor Scheme for Reducing Signalling Costs in Personal Communications Networks", IEEE/ACM Transactions on networking, Vol 4(5), 1996

[12] ITU-T Recommendation G.114, One-way transmisson time, 2003. 